\title{
CONSTRUÇÃO DA PRESCRIÇÃO DE ENFERMAGEM INFORMATIZADA EM UMA UTI
}

\author{
[Building of a computerized nursing prescription for an ICU]
}

\author{
Daise Ribeiro Aquino* \\ Wilson Danilo Lunardi Filho**
}

RESUMO: O Processo de Enfermagem (PE) contribui para a consolidação da profissão como ciência do cuidado. Esse estudo descreve uma construção coletiva da Prescrição de Enfermagem Informatizada (PEI), enfocando os protocolos de intervenções. O objetivo é apresentar o modo de construção coletiva de um instrumento metodológico para o trabalho, a partir do conhecimento da realidade e da interação dos indivíduos. Sugere um modelo simplificado para operacionalizar o PE, buscando atender as necessidades tanto da academia quanto das instituições hospitalares, através da aproximação teórico-prática e da atenção à integralidade preconizada pelo Sistema Único de Saúde (SUS). Traça paralelos entre Sistematização da Assistência de Enfermagem (SAE) e PE, bem como a sua complementação na fusão de ambos, dentro da organização do trabalho. O modelo de PE, fundamentado na teoria de Wanda Horta, constituiu-se de três fases: histórico, evolução e prescrição informatizada. $O$ acesso às intervenções no sistema informatizado acontece pela escolha do(s) problema(s) de enfermagem, dentro de subsistemas corporais afetados. A abordagem metodológica utilizada foi a pesquisa convergente-assistencial com a equipe de

*Enfermeira, aluna do Curso de Mestrado em Enfermagem do Programa de Pós-Graduação em Enfermagem da Fundação Universidade Federal do Rio Grande - PPGEnf/FURG, Membro do Núcleo de Estudos e Pesquisas em Saúde - NEPES/FURG.

**Enfermeiro, Prof. Adjunto IV do Departamento de Enfermagem da Fundação Universidade Federal do Rio Grande - DEnf/FURG, Doutor em Enfermagem, Docente do Curso de Mestrado em Enfermagem do Programa de Pós-Graduação em Enfermagem da Fundação Universidade do Rio Grande - PPGEnf/FURG, Membro do Núcleo de Estudos e Pesquisas em Saúde - NEPES/FURG, Orientador. enfermagem da UTI de um Hospital Universitário, que seguiu os seguintes passos para a construção dos protocolos e implantação da PEI: conhecimento da realidade, pela observação sistemática e pesquisa documental; construção individual dos protocolos, com a consulta bibliográfica; construção coletiva, através da estratégia de revisão dos protocolos pelos enfermeiros. Como resultado obtiveram-se 177 intervenções para 64 problemas. O estudo evidencia a viabilidade da adoção do PE e uso da PEI como fundamentais para a valorização e organização do trabalho da enfermagem.

PALAVRAS-CHAVE: Processo de Enfermagem; Construção Coletiva; Prescrição Informatizada; Protocolos de Intervenções.

\section{RAZÕES PROPULSORAS DO MOVIMENTO EM BUSCA DA CONCRETIZAÇÃO DA PRESCRIÇÃO DE ENFERMAGEM INFORMATIZADA}

O ser humano, pertencente a uma espécie dotada de raciocínio e organizada em sistemas gradualmente complexos de relações, estabelece uma forma de comunicação entre o mundo interno e externo, ou seja, dele como o meio, resultando em produto para ambos, denominado trabalho. $\mathrm{Na}$ ação no mundo através do trabalho, o ser humano formou relações cada vez mais complexas e estruturas sociais que foram sendo consolidadas no produto, configurando a estruturação do capitalismo.

Seguindo a necessidade de expansão e busca de conhecimento, construiu também um 
mundo globalizado, usando a tecnologia. Dentro desse contexto, os valores resultantes dessa caminhada se expressam na forma de considerar o produto do trabalho trocado por capital, virtual ou real, como o objetivo-fim da ação e a vida, essência dos seres, como meio. Portanto, num mundo onde a vida possui um valor questionável, quando considerada como meio para obtenção do produto, as profissões que realizam seu trabalho voltado para o seu cuidado são influenciadas por essas estruturas ideológicas sócio-históricoculturalmente construídas.

O trabalho da enfermagem aparece nesse cenário como uma força de reação a favor da vida, em especial da vida humana, assumindo a roupagem do cuidado. Para um trabalho que apresenta múltiplas dificuldades em seu exercício, a organização é uma maneira de montar estratégias de enfrentamento dessa realidade. Entre as muitas formas de organizar o trabalho da enfermagem, o Processo de Enfermagem (PE) apresenta-se como um modelo de ação capaz de auxiliar no cuidado.

No presente estudo, propomos um modelo de PE simplificado, fundamentado na teoria de Wanda Horta (1979), o qual se constitui de etapas explícitas e implícitas. As primeiras são aquelas possíveis de comprovação pelos registros de enfermagem: o histórico, a prescrição e a evolução, contidos no prontuário do paciente. Por sua vez, a detecção dos problemas de enfermagem para a realização da prescrição de enfermagem pertence ao grupo das etapas implícitas, por fazer parte dos processos mentais de raciocínio lógico necessários ao desempenho destas atividades.

O PE utiliza um histórico de enfermagem constituído por quatro questões norteadoras; faz a identificação e explicitação de problemas (ao invés de diagnósticos de enfermagem); dá origem à Prescrição de Enfermagem Informatizada (PEI), com intervenções objetivas; realiza uma evolução concisa. Deu mostras de ser possível de ser aplicado tanto na academia quanto na prática de enfermagem, apresentando resolutividade no atendimento de necessidades de saúde presentes, durante a internação hospitalar.

Sistematizar é organizar informações padronizadas, de forma a construir sistemas operativos para atingir um objetivo. Esses sistemas precisam estar associados e dinamicamente atuantes para que possam dar e receber a sustentação que necessitam para continuarem constantemente operantes. Nesse estudo, consideramos a Sistematização da Assistência de Enfermagem (SAE) como todo o planejamento registrado da assistência que abrange, desde a criação e implantação do manual de normas e rotinas das unidades à descrição padronizada dos procedimentos técnicos até, finalmente, a adoção do PE.

Em outras palavras, para a execução do $P E$, é fundamental existir a SAE, uma normatização prévia das ações que estarão contidas na PEl. Além disso, o PE também auxilia no cumprimento das normas e rotinas da SAE. Sem uma assistência organizada, padronizada, ou seja, sistematizada, torna-se muito difícil o uso da PEI.

Um trabalho organizado e sistematizado pode demonstrar a força existente na categoria profissional da enfermagem em produzir novos saberes, dirigir e planejar com autonomia o seu fazer. Por outro lado, o condicionamento do seu trabalho à prescrição de outra categoria profissional traduz uma crise de identidade profissional, sendo necessária e urgente a construção de novos conhecimentos que configurem independência e autonomia para a enfermagem.

Partindo dessas considerações, com a realização desse estudo tivemos como objetivo geral construir coletivamente um instrumento metodológico para o trabalho da enfermagem, a partir do conhecimento da realidade e da interação dos profissionais que constituem a equipe de enfermagem para a operacionalização do PE proposto. Para tanto, buscamos atingir os seguintes objetivos específicos:

1. identificar os problemas de enfermagem mais freqüentes apresentados por pacientes de uma UTI Geral;

2. compilar as intervenções recomendadas na literatura para os problemas identificados, adaptá-las à realidade da UTI Geral e inseri-las no banco de dados do Sistema de Apoio à Decisão no Planejamento e Prescrição de Cuidados de Enfermagem (SAD-PPCE), para a elaboração de protocolos assistenciais de enfermagem. 
2 A CONSTRUÇÃO DA PRESCRIÇÃO DE ENFERMAGEM INFORMATIZADA NA PRÓPRIA CAMINHADA

A participação dos seres humanos nos processos de transformação do mundo em que vivemos ocorre por meio da construção contínua do conhecimento, conduzindo a melhorias e à evolução. Essa construção é plural e, mesmo quando realizada em espaços individuais, estamos inseridos em uma rede de relações com os outros e cada ação reflete em repercussões externas ao indivíduo, sendo o mundo, portanto, uma construção coletiva.

Para tanto, é importante entender o modo como os indivíduos constroem o mundo em que vivem, de forma holística, seguindo os princípios de valorização das experiências prévias dos participantes, da interação social para a construção do conhecimento e a relevância dos esquemas individuais de percepção da realidade. Assim, para desenvolver o método de trabalho proposto, a partir de uma prática assistencial, foi necessário construílo juntamente com todos aqueles envolvidos no processo e que seriam os responsáveis pela sua execução. Para tanto, realizamos um estudo do tipo convergente-assistencial, que propõe, a partir do reconhecimento da realidade local, a sua modificação coletiva, resultante da soma de esforços de todos.

A pesquisa convergente-assistencial caracteriza-se como "um trabalho de investigação, porque propõe refletir a prática assistencial a partir de fenômenos vivenciados no seu contexto", podendo incluir construções conceituais inovadoras. Esse tipo de estudo mantém uma estreita relação com a situação social, articulando a prática com o conhecimento teórico, canalizando seus resultados para a solução de problemas existentes nas situações práticas (TRENTINI; PAIM, 1999, p. 27).

O presente estudo foi desenvolvido na Unidade de Terapia Intensiva do Hospital Universitário Dr. Miguel Riet Corrêa Júnior da Fundação Universidade Federal do Rio Grande (HU-FURG). Para a construção da prescrição de enfermagem, foi necessário o envolvimento dos sujeitos no aperfeiçoamento dos protocolos assistenciais de enfermagem, ou seja, enfermeiros, auxiliares e técnicos de enfermagem da UTI do HU. A participação desses profissionais tornou-se fundamental, principalmente, pela sua decisão na elaboração, no caso dos enfermeiros, e interpretação e execução, no caso dos auxiliares e técnicos de enfermagem.

Em respeito aos princípios éticos, os sujeitos foram esclarecidos, através do convite de participação, acerca do objetivo e metodologia do trabalho, sendo garantido ainda o sigilo que assegura a privacidade individual e coletiva da equipe, quanto aos dados confidenciais envolvidos na pesquisa, bem como em devolver-lhes os resultados do estudo, tão logo findasse. Foi solicitado aos entrevistados que assinassem o Consentimento Livre e Esclarecido, ressaltando que, se durante o estudo, por algum motivo, sentiam-se impossibilitados de continuar participando, seria garantida a todos a possibilidade de desistência a qualquer momento.

\section{PASSOS NA ELABORAÇÃO DOS PROTOCOLOS ASSISTENCIAIS: MÉTODO DE COLETA, REGISTRO E ANÁLISE DOS DADOS}

A coleta de dados começou com a observação sistemática dos problemas de enfermagem apresentados por pacientes internados na unidade, utilizando uma planilha para o registro dos dados. Na observação sistemática, "pode-se observar eventos no contexto em que se dão e observar a relação com outros atos e as circunstâncias que influenciaram a sua ocorrência e permitir uma análise mais compreensiva dos dados". A análise dos eventos observados deve produzir descrições que se fundamentem na freqüência das incidências (CHIZZIOTTI, 1998, p. 53). Como esta forma de investigação não enfocou os estados de saúde de seres humanos em particular, nem sequer busca agora divulgá-los, houve o entendimento de ser desnecessária a solicitação do consentimento daqueles que os apresentavam, uma vez que as intervenções de enfermagem daí derivadas poderiam ser aplicadas universalmente a outros pacientes internados ou que internarem nesta mesma unidade ou em outras unidades com características semelhantes.

A busca pela identificação de problemas durou uma semana, alternando a observação do 
exame físico diário e da dinâmica de trabalho local com a pesquisa documental. A pesquisa documental visou utilizar informações documentadas sobre os problemas apresentados pelos pacientes e que iam ao encontro dos objetivos desta pesquisa, pois foram estes que determinaram que tipo de documentos convinha reunir e analisar (CHIZZIOTTI, 1998). Assim, foram consultados a evolução de enfermagem, a prescrição médica, o livro de intercorrências da enfermagem, o prontuário do paciente e a planilha geral dos problemas.

Concluída essa etapa, passamos para a consulta bibliográfica das intervenções de enfermagem possíveis de serem realizadas para os problemas detectados. A proposta inicial era formar grupos de pesquisa com os enfermeiros, mas foi inviável, devido à indisponibilidade destes profissionais, pois muitos trabalham em dois empregos e em turnos diferentes, ficando difícil reunir todos. Essa etapa durou um tempo maior do que o esperado, devido à variedade de informações encontradas e também porque houve dificuldade em encontrar intervenções de enfermagem para alguns problemas na bibliografia disponível.

Visando adaptar as intervenções sugeridas na literatura com as presentes na realidade da unidade e ainda acrescentar outras que eram realizadas, usamos como estratégia para obtenção de dados a revisão pelos enfermeiros, do conteúdo dos protocolos elaborados, com o intuito de validá-los. Além de uma estratégia para obtenção de dados, foi também uma alternativa de aproximar a teoria da realidade do contexto da prática, tão fortemente marcada nesse tipo de pesquisa convergente-assistencial.

$A$ análise foi realizada juntamente com a coleta de dados, corroborando Trentini e Paim, quando dizem que a coleta de dados ocorre simultaneamente à análise. Neste estudo, todas as cinco fases de construção da PEI foram permeadas por esse princípio. A organização das informações facilitou a codificação, ou seja, o reconhecimento das palavras, frases, parágrafos ou temas-chave, que persistem nos dados. Para identificar códigos, é preciso ler, reler, refletir sobre as informações, a fim de unir informações comuns relatadas pelos participantes, para posteriormente formar categorias, concluindo o processo de análise (TRENTINI e PAIM, 1999, p.104-105).

\section{Primeiro passo - Aproximação com a realidade}

O período de observação foi imprescindível, já que nossa inserção na realidade local foi apenas pela ligação com a pesquisa. Frente a esse problema,

\begin{abstract}
é oportuno salientar que se torna quase impossível conduzir uma pesquisa do tipo convergenteassistencial sem ter familiaridade com o contexto social em estudo, pois este objetiva introduzir mudanças no ambiente; nada impede que um pesquisador externo (que não atua na área) desenvolva este tipo de pesquisa, desde que se insira na prática assistencial do local escolhido, e para isso, o pesquisador necessitará, evidentemente, de tempo e paciência somados à habilidade de se fazer aceito pelo pessoal interno (TRENTINI; PAIM, 1999, p. 152).
\end{abstract}

A busca dos problemas através da observação sistemática e da pesquisa documental resultou em 83 problemas identificados. Isto porque a consulta a evoluções de enfermagem de até quatro dias anteriores tornou possível ampliar a gama de problemas identificados, indo além daqueles coletados apenas na situação atual dos pacientes no dia da coleta. A consulta à prescrição médica serviu não apenas para identificar as medicações mais usadas, mas evidenciou que, muitas vezes, também contempla a prescrição de intervenções de enfermagem, o que poderia futuramente ser modificado com a adoção definitiva da própria prescrição de cuidados de enfermagem (no período de experimentação nenhuma mudança nesse sentido foi observada).

Para partir em direção à consulta bibliográfica, tornou-se necessário agrupar os problemas de acordo com a similaridade apresentada na intervenção recomendada pela literatura, pois se o número de problemas e a repetição de intervenções forem reduzidos, facilita a construção dos protocolos. Por exemplo, os problemas sudorese, sialorréia (salivação excessiva), halitose, secreção nasal foram aderidos ao problema: higiene precária, pois seu protocolo de intervenções é amplo e os contempla. Houve alguns itens que acabaram sendo excluídos por exigir, predominantemente, a intervenção medicamentosa ou não apresentaram uma proposta relevante de ação, que necessitasse estar contida na prescrição de enfermagem da UTI. São eles: taquicardia, cefaléia, uso de antitérmico, de 
anticoagulante, de noradrenalina e de creme para lesões. No final do processo de síntese, dos 83 problemas iniciais, resultaram apenas 64 .

Da síntese, partiu-se para a categorização na classificação e inserção dos problemas em subsistemas. Nessa visão, os problemas foram identificados dentro dos subsistemas corporais afetados, conforme acontece durante o exame físico diário. Nesse sentido, pôde-se concluir que o modelo de PE proposto, embora tenha seus princípios na teoria de Horta, classificou os problemas de enfermagem dentro de subsistemas corporais, ao invés das necessidades humanas básicas afetadas.

Os subsistemas foram associados, formando cinco grandes categorias: Cardiorrespiratório, Gastroentérico, Geniturinário, Neuromotor/Pele e Terapêutica. Os subsistemas cardíaco e respiratório foram agrupados pela relação fisiológica de interdependência entre um e outro na manutenção da vida. Já os subsistemas genital e urinário foram agrupados pela localização anatômica, que também facilita a detecção de sinais e sintomas, durante o exame físico. Os sistemas nervoso, motor e a pele foram associados por causa e conseqüência já que, quando há comprometimento do sistema nervoso, esse afeta diretamente os músculos e também a pele, como no caso do coma que resulta em imobilidade e possível tendência à formação de úlceras de decúbito.

Ainda, tornou-se necessário formar a categoria da Terapêutica, que não envolve nenhum sistema corporal específico, mas todos ou alternadamente, a depender das drogas utilizadas. Com a multiplicidade de drogas manipuladas na UTI, as intervenções relacionadas aos seus efeitos precisavam dessa classificação especial para aplicar e ampliar os conhecimentos farmacêuticos da enfermagem.

\section{Segundo passo - Construção individual dos protocolos assistenciais de enfermagem}

$\mathrm{Na}$ busca de intervenções de enfermagem para construir os protocolos, utilizamos os achados dos trabalhos acadêmicos realizados na própria UTI do HU-FURG (COSTA, 2003; FERREIRA, 2002), filtrando as informações semelhantes e associando à dissertação de Marcon (2002). Após, consultamos a bibliografia de caráter mais técnico (BRUNNER; SUDDARTH, 1998a, 1998b; GAGLIAZZI, 2000; MENNA BARRETO, 1993; SOARES, 2000), buscando contemplar o conjunto de intervenções almejado. Também, foram utilizados conteúdos acadêmicos aprendidos durante a graduação e o Manual de Rotinas da UTI do HU-FURG (SIGILIO e cols. 2003). Esse manual ainda está em construção, mas permitiu conhecer a divisão de tarefas por cada turno e estabelecer o aprazamento da troca de curativos e materiais do paciente nas intervenções pesquisadas.

A busca resultou em 135 intervenções para os 15 problemas referentes ao Subsistema Cardiorrespiratório, 115 intervenções para os 18 problemas referentes ao Subsistema Neuromotor e Pele, 68 intervenções para os 9 problemas do Subsistema Gastroentérico, 46 intervenções para os 14 problemas do subsistema Terapêutico e 13 intervenções para os 3 problemas do Subsistema Geniturinário, totalizando 377 intervenções de enfermagem, embora algumas dessas intervenções repetirem-se para diferentes problemas.

Acatando sugestões (do professor orientador), foi modificada a forma de registro de problemas - como no caso de "catéter nasal", passou a ser "oxigenoterapia por catéter nasal" para, só a partir daí poder ser considerado como um problema apresentado pelo paciente e que, para sua resolução, necessitava de intervenções de enfermagem. Outra sugestão foi a retirada de intervenções que correspondiam à mera descrição de procedimentos técnicos como, por exemplo, "abrir a SNG 15 minutos, antes da administração da dieta", reduzindo-se, assim, a 346 intervenções.

Depois de reunidas, as 346 intervenções foram aprazadas, de acordo com o preconizado na literatura e com a rotina da unidade, de maneira a garantir a operacionalização da prescrição em uma escala de tempo determinada. Visando encontrar uma forma de aproximar as recomendações da literatura com o contexto prático, os prazos de $3 / 3 \mathrm{~h}$ foram adaptados para serem realizados duas vezes por turno, proporcionando maior flexibilidade na sua execução. O mesmo aconteceu com os 
procedimentos a serem realizados três vezes ao dia, que passaram a ser estipulados uma vez por turno, recebendo o código: $\mathrm{M}, \mathrm{T}, \mathrm{N}$ - representado o turno da manhã, da tarde e da noite, respectivamente, a ser checado logo após sua execução.

Os aprazamentos com hora fixa $8 / 8 \mathrm{~h}(08 \mathrm{~h}-$ $16 \mathrm{~h}-24 \mathrm{~h})$ ou $6 / 6 \mathrm{~h}(06 \mathrm{~h}-12 \mathrm{~h}-18 \mathrm{~h}-24 \mathrm{~h})$ mostraram-se mais adequados para as intervenções existentes no subsistema terapêutico, uma vez que as intervenções relacionadas aos medicamentos precisam ser realizadas, antes ou após o horário da administração. As intervenções que são realizadas apenas uma vez por dia $(1 \mathrm{x} /$ dia) são executadas, conforme a rotina de divisão das tarefas entre os turnos. Na UTI Geral do HUFURG, os procedimentos executados $1 \mathrm{x} / \mathrm{dia}$ com os pacientes que estivessem nos leitos 01 e 02 , eram realizados no período da manhã, nos leitos 03 e 04, à tarde, e nos leitos 05 e 06, à noite.

Intervenções mais intensivas receberam os prazos de $h / h$ e $2 / 2 h$. Rotinas de trocas de materiais e manutenção receberam, em sua maioria, os prazos de $72 \mathrm{~h}$ ( $3 / 3$ dias), 48h ( $2 / 2$ dias), $1 \mathrm{x} /$ semana, 20 dias e 60 ou 80 dias. Alguns aprazamentos atípicos determinaram um limite de prazo admitido para a realização de determinado procedimento, como quando se solicita a realização da intervenção em $2 \mathrm{~h}$, em $4 \mathrm{~h}$, em $5 \mathrm{~h}$, em 6h, em 24h, em 7 dias e "amanhã".

Embora a literatura discrimine que a prescrição deverá ser válida por 24 horas, em algumas intervenções o aprazamento pode ser maior, continuando a descrição da data nas próximas prescrições. Isso acontece porque as rotinas de troca são admitidas como intervenção de enfermagem, uma vez que previne infecção e lesão no paciente. A troca do curativo do Intracath é exemplo: realizado em 48 horas, recebendo a data do dia de sua próxima troca.

As intervenções que são realizadas somente quando um determinado evento ocorre, recebem o aprazamento "QN", ou seja, quando necessário. Isso significa a sua validação somente no momento em que o profissional julgar como necessário, fato que ocorre, por exemplo, nas intervenções "aspirar secreções traqueo-brônquicas, registrando cor, volume, consistência e odor", "estimular a evacuação com supositório de glicerina" e "registrar
ECG, durante episódio de dor torácica". Ao ser checada, a intervenção deverá conter a hora da sua realização, facilitando assim, o registro das intercorrências, a comunicação escrita entre os turnos e o fácil fluxo de informações como a hora do infarto, o tempo de acúmulo das secreções traqueo-brônquicas e a freqüência do uso de supositórios.

Outro fato interessante ocorre na mudança de decúbito, contendo as quatro posições mais usadas na UTI do HU (DLE, DD, DLD e Fowler), seguindo as recomendações de Horta (1979, p. 67) que diz, "quando for prescrita mudança de decúbito, deverão ser explicitadas as posições indicadas com os respectivos horários." $\mathrm{Na}$ prescrição, são descritas doze vezes, a fim de serem checadas e contempladas no período de 24h: DLE - DD - DLD - FOWLER - DLE - DD DLD - FOWLER - DLE - DD - DLD - FOWLER. Existe também o aprazamento para as intervenções realizadas próximo a cada refeição, ou seja, no café da manhã, no almoço, no café da tarde, na janta e no lanche, representado por "CA-C-J-L".

Outra etapa transposta e também importante na construção da prescrição foi a escolha minuciosa dos verbos que determinam as ações prescritas. O conjunto de verbos utilizado inicialmente foi: avaliar, aspirar, aplicar, aquecer, atentar, aerar, administrar, afrouxar, apoiar, aumentar, conferir, cobrir, comunicar, coletar, colocar, cortar, conter, desativar, desprezar, desobstruir, desinflar, detectar, evitar, estimular, encaminhar, elevar, esvaziar, fornecer, fluidificar, hidratar, implementar, incentivar, instituir, infundir, informar, higienizar, monitorizar, manter, medir, ordenhar, oferecer, posicionar, preparar, proteger, proporcionar, providenciar, registrar, realizar/ orientar/auxiliar, retardar, remover, trocar, tracionar, testar e verificar. Essa multiplicidade de verbos e diferentes intervenções com o mesmo significado ocorreu por serem usadas bibliografias variadas na construção das intervenções (BRUNNER; SUDDARTH, 1998a, 1998b; GAGLIAZZI, 2000; MENNA BARRETO, 1993; SOARES, 2000). Após a análise do significado e aplicação de cada verbo, foram sendo sintetizados e reduzidos de 54 para apenas 20 verbos diferentes.

Para elucidar melhor esse processo, têm-se exemplos de alguns verbos que foram substituídos por outros, a fim de posteriormente facilitar uma pretensa classificação das ações de enfermagem à luz da teoria de Horta. Os verbos revisar, garantir 
e conferir foram trocados por verificar; o verbo avaliar, quando relacionado à objetos, também foi substituído por verificar, ficando reservado à avaliação apenas as intervenções relacionadas ao paciente; da mesma forma, os verbos investigar, inspecionar e vigiar foram substituídos por avaliar; as ações de controle e monitorização receberam o verbo monitorar; lavar e limpar foram substituídos por higienizar; promover e proporcionar por estimular; umidificar e gotejar por hidratar, massagear, ordenhar e cortar por realizar. Exemplos:

1. avaliar a manutenção do selo d'água no frasco e a constante oscilação do líquido (verbo incorreto).

2. verificar a manutenção do selo d'água no frasco e a constante oscilação do líquido (verbo correto).

Um verbo muito encontrado nas prescrições e na bibliografia foi "observar". Evitou-se a sua utilização nesse modelo, porque na observação, a ação se restringe à constatação de uma informação. Entretanto, na avaliação, espera-se que o profissional além de constatar, processe essa informação de maneira a transformá-la em uma outra ação. Então, optou-se por substituir o verbo observar por avaliar ou verificar.

Nesse modelo, o uso da classificação dos verbos por grau de dependência não foi adotado, pois entende-se que a natureza da assistência já está expressa no próprio verbo que inicia a intervenção. Quando o objetivo é, por exemplo, movimentar os membros do paciente, o profissional que cumprirá a prescrição irá avaliar as condições do paciente. Se o paciente puder realizar sozinho, ele deve apenas estimular, se precisar ser ajudado, deve auxiliar e se precisar que faça por ele, o verbo é realizar. Assim, seguem outros exemplos de como as intervenções foram construídas, visando promover essa flexibilidade:

1. realizar/orientar massagem circular abdominal, da direita para a esquerda;

2. realizar/auxiliar higiene oral com solução antisséptica.

Essa flexibilidade oferece maior autonomia a quem executa a prescrição, ou seja, o enfermeiro/ técnico/auxiliar de enfermagem. Com isso, estimula-se a avaliação prévia do paciente, antes de ser instituída a intervenção, justificando o não uso da classificação por grau de dependência, proposta por Horta (1979). Para a autora, o grau de dependência pode ser total, que compreende tudo que a enfermagem faz pelo ser humano, quando este não tem condições de fazer por si, ou parcial, quando a assistência de enfermagem situa-se em termos de ajuda, orientação, supervisão e encaminhamento.

No final da análise, foi possível classificar verbos quanto à natureza da assistência, reforçando que o grau de dependência pode ser expresso apenas pelo verbo:

- FAZER: Realizar; Administrar; Aspirar; Encaminhar; Esvaziar; Evitar; Fluidificar; Hidratar; Higienizar; Implementar; Manter; Trocar.

- AJUDAR: Auxiliar; Estimular.

- ORIENTAR: Orientar.

- SUPERVISIONAR; Verificar; Monitorizar; Avaliar; Comunicar; Registrar.

Segundo Horta (1979, p. 67), "a prescrição precisa ser concisa, clara e específica". Ao serem selecionados problemas, estes devem conter intervenções sintetizadas e concisas, evitando prescrições extremamente longas e detalhadas. Partindo dessas premissas, uma preocupação na sua construção foi analisar cada uma das intervenções, buscando repetições e informações semelhantes encontradas nas bibliografias. Assim, algumas intervenções foram agrupadas pela similaridade das ações e por possuírem o mesmo aprazamento.

Frente às 346 intervenções, a exclusão e simplificação de muitas delas foi um avanço na sua construção. Escolheu-se, entre elas, as que representavam com mais clareza para os profissionais a solicitação prescrita. Algumas representantes dessa nova etapa de síntese foram as intervenções: entre "desobstruir e higienizar vias aéreas superiores" e "aspirar secreções de oro e nasofaringe", optou-se pela segunda; entre "manter cabeceira elevada" e "posicionar paciente em FOWLER alta $\left(90^{\circ}\right)$ ", optou-se pela primeira; entre "oferecer líquidos com freqüência em pouco volume" e "estimular ingesta hídrica de 2-3 litros ao dia, em pequenas porções ( $200 \mathrm{ml}$ ou um copo) $-2 / 2 h$ ", optou-se pela segunda, principalmente por ser mais específica e detalhada.

Outra alternativa encontrada foi o "protocolo de rotinas", agrupando em uma só intervenção, 
todas as demais. O problema "contenção mecânica" recebeu como intervenção: "Implementar protocolo de rotinas para contenção mecânica: verificar circulação sanguínea nas extremidades, afrouxar contenção e exercitar os membros e extremidades - 2x/turno". Além desse, também foram formados protocolos de rotinas para: desmame do respirador, uso de sonda nasoentérica, recuperação do tônus vesical, hemoterapia, úlcera de decúbito, óbito, préoperatório, adaptação à colecistectomia e à gastrostomia. Com isso, ficou mais próximo o objetivo de construir prescrições concisas, através da redução e simplificação das intervenções.

\section{Terceiro passo - Construção coletiva dos protocolos assistenciais de enfermagem}

Foi entregue a cada um dos cinco enfermeiros, o material contendo os protocolos elaborados, de acordo com o subsistema corporal (gastroentérico,

neuromotor/pele, cardiorespiratório, genitouninário ou terapêutica) de sua escolha, para realizar a revisão escrita das intervenções. Todos tiveram acesso aos materiais distribuídos aos demais, pois eram passados nos plantões para serem entregues a nós. Demonstraram muito entusiasmo e boa receptividade à proposta e entregaram, em média, após uma semana. Essa etapa resultou na modificação de aproximadamente 50 intervenções, incluindo algumas exclusões, inclusões, correção das medidas e aprazamentos, além da adaptação à linguagem escrita da unidade.

Mesmo com a modificação escrita dos enfermeiros, ainda suscitavam dúvidas em relação ao correto aprazamento e a real funcionalidade das intervenções, pois nas revisões emergiram sugestões diferentes entre os enfermeiros. Após um novo processo de revisão, realizado através de uma reunião com o enfermeiro chefe da unidade, foram identificadas as intervenções que apenas reproduziam parte da rotina diária, o que abalaria a credibilidade da prescrição de enfermagem, se inseridas no sistema. Após mais essa minuciosa seleção, contemplou-se finalmente o conjunto de 176 intervenções.

As 176 intervenções foram cadastradas no sistema informatizado (SAD-PPCE) e após relacionadas com os respectivos problemas de enfermagem, em outro campo do programa. Cada um dos 64 problemas recebeu um código, de modo a facilitar a busca pelos enfermeiros, sendo codificado como "GAS", os problemas existentes no sistema gastroentérico, "CAR", os existentes no cardiorrespiratório, "NEU", os existentes no sistema neuromotor e pele, "GEN", os existentes no genitourinário e "TER", os problemas da terapêutica. Todos os enfermeiros foram orientados sobre o manuseio do programa e referiram que os códigos facilitaram o acesso aos problemas e às intervenções. Além disso, os dados dos enfermeiros e dos pacientes foram cadastrados previamente, pois o SAD-PPCE permite a entrada nas prescrições somente após o seu fornecimento.

Após essas fases de construção, a implantação ocorreu com a elaboração da prescrição de enfermagem pela enfermeira e a pesquisadora diariamente, no turno da noite, antes das $22 \mathrm{~h}$, prazo limite de validade da prescrição anterior. Essa etapa teve duração de aproximadamente quinze dias. Nesse período, foi possível que as rotinas se concluíssem e se repetissem, possibilitando uma maior evidência de todo o processo. A meta atingida foi o atendimento de todos os pacientes que internaram na unidade, através do uso da PEI. No final de cada período de observação participante, foram realizados registros diários acerca das percepções e sugestões dos participantes para a PEl.

Pôde-se perceber que a prescrição teve boa aceitação na rotina de trabalho desses profissionais, pois era checada diariamente e houve poucos episódios de esquecimento. Os auxiliares/técnicos na sua maioria, seguiram e mantiveram a PEI checada, entregando-a na passagem de plantão. Entretanto, nenhuma prescrição foi realizada novamente pelos enfermeiros de outros turnos, quando houve intercorrências.

Além disso, foi muito gratificante a participação dos profissionais, em especial dos auxiliares de enfermagem do turno da tarde, que sempre traziam novas sugestões para que a prescrição pudesse ser melhorada. Entre elas, cito algumas principais:

1. a prescrição é muito extensa, deve-se tentar resumí-la, principalmente quando descreve vários sinais e sintomas que precisam ser avaliados no paciente;

2. conter campos para serem registradas 
com um "X" as alterações apresentadas pelo paciente durante o plantão, como convulsões, diarréia, sudorese, taquicardia;

3. deveria ter o aprazamento preenchido pelo profissional que executa, ao invés de já vir aprazada, pois pode haver mudanças durante o plantão, como a troca do intracath e corte de unhas antes do prazo prescrito;

4. aprazar mais vezes a higiene oral e a troca de decúbito à noite, pois o turno tem duração de 12 horas e, quando é 2x/turno, na noite deve ser realizado quatro vezes.

Como solução para a sugestão 1 e 2, utilizouse fazer a checagem em cima da palavra que corresponde ao sinal ou sintoma apresentado pelo paciente, identificando o turno ou o horário que aconteceu, da seguinte forma: convulsões $/ M / \mathrm{T} / \mathrm{N}$. Com esse controle, as alterações apresentadas pelos pacientes são registradas a fim de facilitar o fluxo de informações intra-equipes de enfermagem, bem como com as equipes médica, de fisioterapia e outras.

A sugestão 3 foi ouvida e (re)sugerido que fosse checada a intervenção, mesmo num período precoce, porém deveria ser registrada acima da checagem, a data ou o horário realizado para que, na próxima prescrição, essa data fosse considerada e o novo aprazamento ocorreria a partir dela. A sugestão 4 foi auxiliada por um enfermeiro que explicou ao participante sobre a inviabilidade de movimentar os pacientes à noite para a realização de procedimentos.

A prescrição de enfermagem informatizada entrou facilmente na rotina da unidade, ficava na prancheta do paciente, juntamente com a folha de balanço hídrico e prescrição médica. Ao iniciar uma prescrição de enfermagem informatizada nova, a antiga ficava arquivada em uma pasta de documentos do paciente, onde também estavam as evoluções antigas. Alguns auxiliares e técnicos relataram que as checavam somente no final do plantão, após fecharem a folha de balanço hídrico parcial. Observou-se também que os participantes realmente as adotaram como método de trabalho e inclusive as utilizavam para fazer registros nas intercorrências.

Ao final desses passos pode-se perceber que emergiu, durante os processos de investigação, as características dos sujeitos, a maneira como criam, modificam e interpretam a realidade. Essa trajetória de construção ocorreu em espaços individuais e coletivos, configurando a proposta da pesquisa convergente-assistencial na dança de aproximação da teoria com a prática da enfermagem, emergindo daí, as concepções dos sujeitos, com suas resistências e possibilidades na adoção da PEl.

\section{PROJEÇÕES FUTURAS PARA O PROCESSO DE ENFERMAGEM}

Além de possíveis mudanças vindas com a adoção definitiva da PEI na UTI, a sua constante reformulação precisará seguir princípios básicos que estabeleçam diretrizes para a construção de futuras prescrições, mesmo que dentro de um modelo que se proponha a ser simplificado:

1. ter como foco a satisfação das necessidades psicobiológicas;

2. conter intervenções realizáveis em uma escala de tempo determinada;

3. conter parte das rotinas e de alguns procedimentos;

4. abranger restritamente o domínio de atuação profissional da enfermagem; do PE;

5. estimular a realização das demais etapas

6. garantir a troca de materiais para a prevenção de infecção;

7. visar a proteção do cliente e do funcionário;

8. ser clara, concisa, objetiva e educativa.

Com esse estudo constatamos que o PE constituído de três etapas, direcionado à solução dos problemas psicobiológicos, classificado por subsistemas corporais, baseado na SAE e auxiliado pelo uso da PEl é viável de ser implementado e pode ser utilizado tanto na academia quanto no ambiente hospitalar. Acrescida a essa visão está, principalmente, a coragem de experimentar o novo, de estar aberto e acreditar na proposta.

O trabalho minucioso de analisar as intervenções uma a uma, para melhor as conhecer, estudar, interpretar, classificar, escolher o melhor verbo, o melhor aprazamento, buscar a clareza na redação, a concisão, o dinamismo e a adaptação à realidade, também é um trabalho artesanal, onde se constrói, desconstrói, molda, acentua curvas, 
pinta, decora e aprecia, sendo inimaginável essa construção acontecer de outra forma.

\section{5 À GUISA DE CONCLUSÃO}

Ao alcançar o objetivo principal, construir e implantar a $\mathrm{PEI}$, fica o questionamento se o $\mathrm{PE}$ simplificado seria viável em outras unidades de internação e em outros hospitais, pois essa construção não pretende ser soberana e única dentro de uma proposta. É, a partir desse ponto, que surgem outras maneiras de enxergar o tema e conseqüentemente, novas razões propulsoras do movimento em busca de respostas.

As incertezas que nos acompanhavam para propor o uso dessa metodologia, exigiram cada vez mais acreditar no benefício e na utilidade da proposta. Muitas vezes, o desânimo insistia em supor ser a PEI apenas mais um papel com o fim único de tentar demonstrar o valor da profissão. Felizmente, as primeiras tentativas foram correspondidas com uma resposta positiva dos participantes que, sem perceber, nos estimulavam e assim também era recíproco. Pensamos, então, na possibilidade da prescrição de enfermagem ser mais um papel, porém o único realmente de total responsabilidade e privacidade do enfermeiro, uma vez que muitos outros foram e continuam sendo burocraticamente assumidos.

Foi muito satisfatório ouvir: "Que bom que tu chegou! Tenho mais um monte de coisas para te falar da prescrição, hoje!". Cada um reagiu diferentemente às situações: uns mais introspectivos, outros mais curiosos, contudo, realmente puderam receber a denominação de "participante", pois foi assim que se portaram, com muita propriedade.

Embora muitas vezes discordássemos dos enfermeiros, principalmente ao excluírem intervenções por eles consideradas como rotineiras, a construção coletiva proporcionou a chegada a um consenso, pelo menos favorável à maioria. O diálogo e a liberdade de expressão estimulou o grupo a refletir sobre suas condutas na prática do cuidado e perceber que, em conjunto, é possível operar mudanças na realidade, através de reflexões compartilhadas, buscando o despertar de um profissional mais reflexivo e crítico.

Ao criar a questão problema, imaginávamos poder descrever inúmeras técnicas de formulação da prescrição de enfermagem, padrões a serem admitidos e sistematizados. Todavia, o caminho tomou o rumo da descoberta, de maneira muito sutil: a resposta estava na interação com os participantes. A escolha da metodologia convergente-assistencial estimulou essa reflexão crítica, pois oportunizou transformar a realidade, através de ações conjuntas e do reconhecimento do contexto onde se daria a transformação.

Essa construção coletiva constituiu-se numa experiência singular para quem a vivenciou. A oportunidade de participar dessa construção trouxe um aprendizado mútuo a todos. Foi buscada, na revisão, nos encontros, na implantação e nas entrevistas, nada mais valioso do que a interação humana. É essa a essência que traduz o valor de uma profissão: como as pessoas se unem e buscam o crescimento a favor dela, para ela e para si.

ABSTRACt: Nursing Process (NP) contributes for the consolidation of the profession as the science of caring. This study describes the team construction of a Computerized Nursing Prescription (CNP) focusing on intervention protocols. The aim is to present the way that a methodological work instrument has been built, through the reality awareness as well as individuals' interaction. It suggests a simplified model to operate NP, aiming to meet academic as well as hospital needs, by means of approaching theory/ practice and attention to the wholeness claimed by the National Health System (Sistema Único de Saúde/ SUS, in Portuguese). It approaches Systematization of Nursing Practice (SNP) and NP, besides their complementariness in their merge, inside working organization. NP model is grounded by Wanda Horta's theory which entails three steps: history, evaluation and computerized prescription. Intervention access to the computerized system is effected by the selection of nursing problem(s) in affected body subsystems. The methodological approach used was the converging-practical research among the ICU nursing team of a Teaching Hospital, and the following steps were followed in order to build the protocols and implement the CNP: reality awareness, systematic observation and document survey; individual making of protocols, with 
bibliographic review; team building by means of the strategic review of protocols by the nurses. As a result, 177 interventions were obtained for 64 problems. The study evidences the feasibility for the adoption of NP and the use of CNP, which are fundamental for valuing and organizing nursing tasks.

KEYWORDS: Nursing Process; Team Building; Computerized Prescription; Intervention Protocols.

\section{REFERÊNCIAS}

BRUNNER, L. S.; SUDDARTH, D. S. Tratado de Enfermagem médico-cirúrgica. Rio de Janeiro: Guanabara Koogan, 1998.

CHIZZIOTTI, A. Pesquisa em ciências humanas e sociais. 3. ed. São Paulo: Cortez, 1998.

COSTA, E. T. S. da. A sistematização da assistência em enfermagem dentro do contexto social e político voltado à qualificação e valorização do profissional. 2003. 57f. Monografia (Curso de Especialização em Projetos Assistenciais em Enfermagem) - Pós-graduação em Enfermagem, Fundação Universidade Federal do Rio Grande, Rio Grande.

FERREIRA, C. R. Protocolos Assistenciais de Enfermagem: Elementos para a Organização do trabalho em um UTI geral. 2002. 47f. Monografia (Conclusão do Curso de Enfermagem e Obstetrícia) - Departamento de Enfermagem, Fundação Universidade Federal do Rio Grande, Rio Grande.

GAGLIAZZI, M. T.; URASAKI, M. B. M.; GONÇALVES, R. Intervenções de Enfermagem. São Paulo: EPU, 2000.

HORTA, W. A. Processo de Enfermagem. São Paulo: EPU, 1979.

MARCON, L. Uma construção coletiva: Protocolo de Cuidados de Enfermagem dos Pacientes com Traumatismo Crânio-Encefálico Severo Internados em Unidade de Terapia Intensiva. 2002. 121f. Dissertação (Mestrado em Enfermagem) - Centro de Ciências da Saúde, Universidade Federal de
Santa Catarina, Florianópolis.

MENNA BARRETO, S. et al. Rotinas em terapia intensiva. 2. ed. Porto Alegre: Artes Médicas, 1993.

SIGILIO, M. R.; CHAPACAIS, M.; LISBOA, L. Rotinas e técnicas de enfermagem na UTI do Hospital Universitário Miguel Riet Corrêa Jr. Rio Grande: Hospital Universitário Dr. Miguel Riet Corrêa Jr., nov. 2003.

SOARES, N. R. Administração de medicamentos na enfermagem. Rio de Janeiro: EPUB, 2000.

TRENTINI, M.; PAIM, L. Pesquisa em enfermagem: uma modalidade convergenteassistencial. Florianópolis: Editora da UFSC, 1999.
Rua Dr. Laviera, 167 Jardim do Sol

Rio Grande/RS 96216-040 lunardifilho@terra.com.br 